\title{
Relearning in Semantic Dementia: Word retraining programs to help rebuild vocabulary
}

\author{
Sharon Savage $^{1,2}$, Olivier Piguet ${ }^{1,3}$, John R. Hodges ${ }^{1,3}$
}

ARC Centre of Excellence in Cognition \& its Disorders, Sydney, Australia; ${ }^{2}$ Psychology department, University of Exeter, Exeter, UK; ${ }^{3}$ Brain \& Mind Centre, University of Sydney, Sydney, Australia

\section{Background \& Aims}

Semantic dementia (SD) significantly impacts word knowledge, resulting in marked deficits in both spoken and written word retrieval.

- Word retraining has been shown to improve spoken word retrieval in patients with mild through to severe impairments. ${ }^{2-4}$

- Remediation of written retrieval has not been studied. This study aims to:

i) investigate the effectiveness of word retraining for both spoken and written word retrieval.

ii) explore the impact of disease severity on treatment success.

\section{Materials \& Methods}

8 SD patients completed word retraining

\begin{tabular}{|c|c|c|c|c|c|c|}
\hline Patient ${ }^{*}$ & Age & Sex & $\begin{array}{l}\text { Education } \\
\text { (y) }\end{array}$ & $\begin{array}{c}\text { Disease } \\
\text { Duration } \\
\text { (y) }\end{array}$ & $\begin{array}{c}\text { ACE-R } \\
(100)\end{array}$ & $\begin{array}{c}\text { SYDBAT } \\
\text { Naming } \\
(30)\end{array}$ \\
\hline Mild-1 & 69 & M & 15 & 5.5 & 86 & 16 \\
\hline Mild-2 & 62 & $\mathrm{~F}$ & 15 & 6 & 80 & 16 \\
\hline Mild-3 ${ }^{a}$ & 63 & $\mathrm{~F}$ & 11 & 6.5 & 79 & 14 \\
\hline Mild-4 & 62 & $M$ & 13 & 5 & 84 & 10 \\
\hline Mod-1 & 63 & $M$ & 16 & 6.5 & 68 & 8 \\
\hline Mod-2 & 71 & $\mathrm{~F}$ & 16 & 9 & 56 & 8 \\
\hline Severe- $1^{a}$ & 50 & M & 12 & 8 & 57 & 4 \\
\hline Severe-2 & 63 & M & 11 & 6.5 & 49 & 2 \\
\hline
\end{tabular}

severity was categorised by performance on a semantic battery

indicates predominately $R>L$ anterior temporal lobe atrophy on MRI

\section{Word Retraining Procedure}

Stimuli: 3 matched lists $\times 30$ photographs of objects + word (visual + audio presentation); 2 trained lists; 1 untrained list Delivery: home computer Method: "Look, Listen, Repeat" 5 times/ week $x 4$ weeks

Assessment Measures

Weekly assessments were conducted throughout:
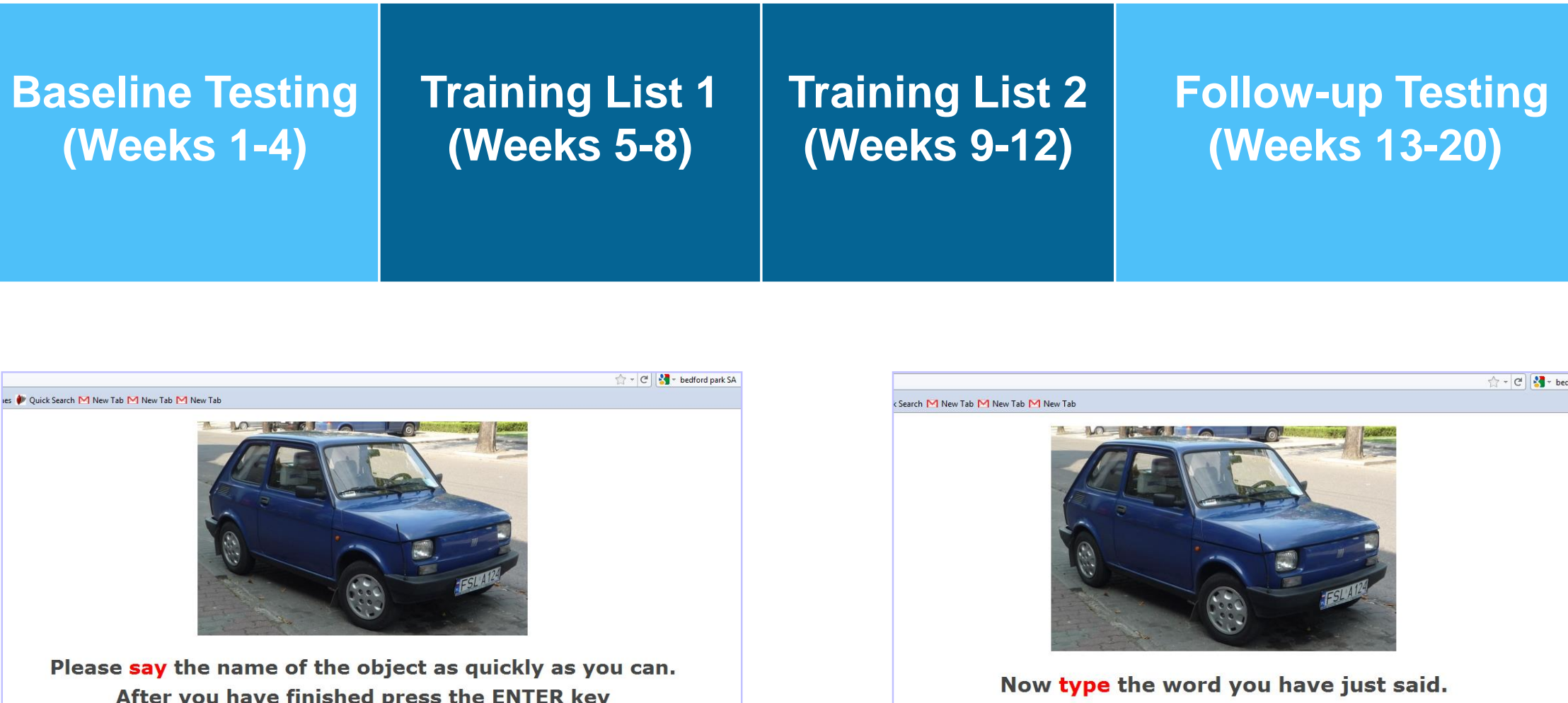

- Naming accuracy: \% words correctly spoken when shown each picture (scored $1=$ correct word; $0=$ incorrect word/ incorrect pronunciation of word);

Written accuracy: \% words correctly typed when shown each picture (scored $1=$ correct word with correct spelling; 0 = incorrect word and/or incorrect spelling);

Error type: frequency of different incorrect responses (categories : i) 'don't know'/ no response; ii) correct word but spelt incorrectly; iii) semantic error - related word or description; iv) other error - unrelated word; or v) neologism (non-word).

\section{Results - Spoken retrieval}

- All participants significantly improved on naming trained words:

Learning: accuracy of naming increased for people with mild, moderate \& severe impairments.

Maintenance: improvements were maintained at the 2-month follow-up compared to baseline.

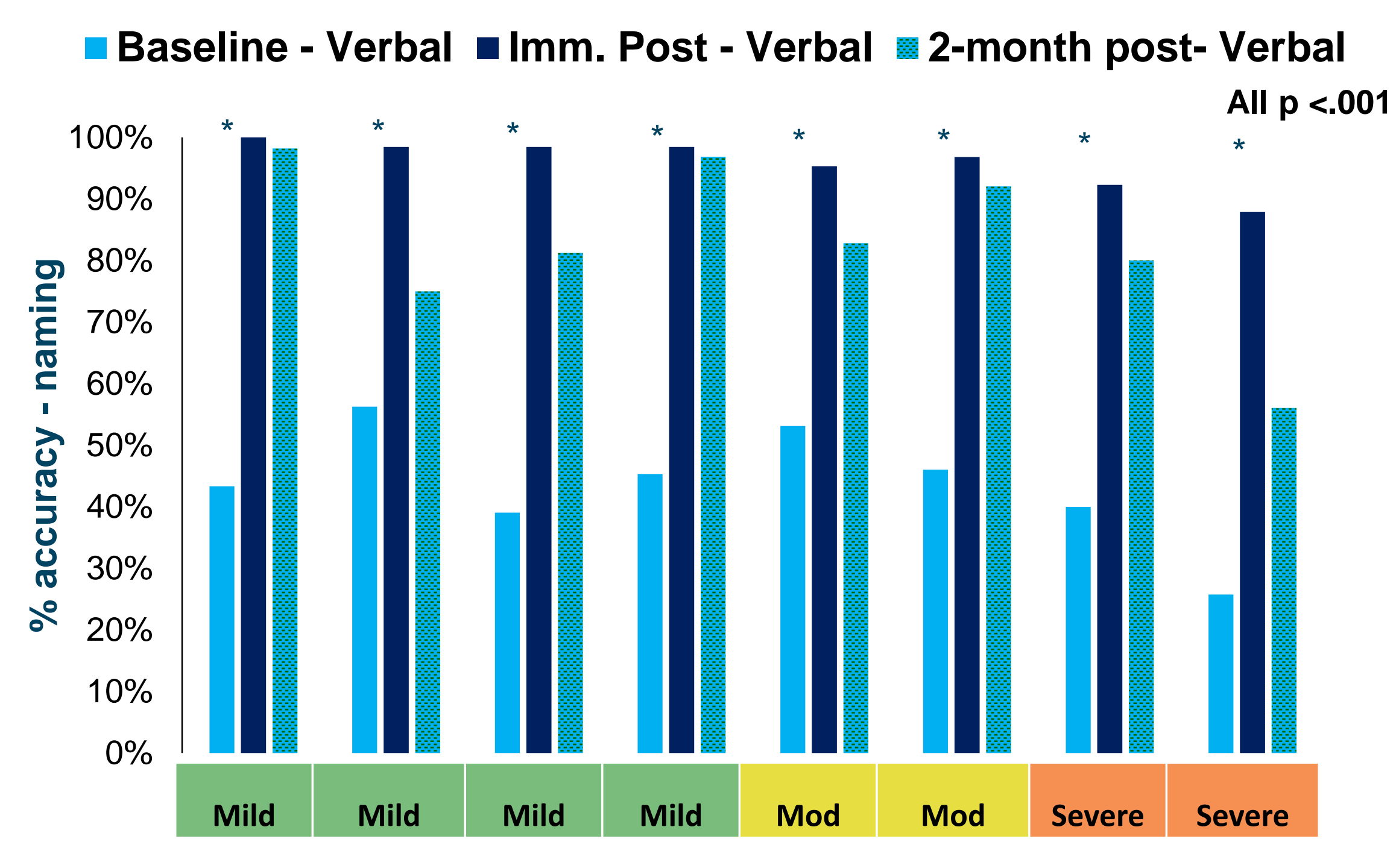

\section{Results - Written retrieval}

- Significant improvements were also seen when writing trained words:

- Learning: written accuracy increased for people with mild, moderate \& severe impairments. mprovements in writing were similar in magnitude to those achieved for spoken retrieval, with the exception of two cases (Mild-2 and Severe-2), where the change in written accuracy was smaller.

- Maintenance: improvements were again well maintained at the 2-month follow-up.

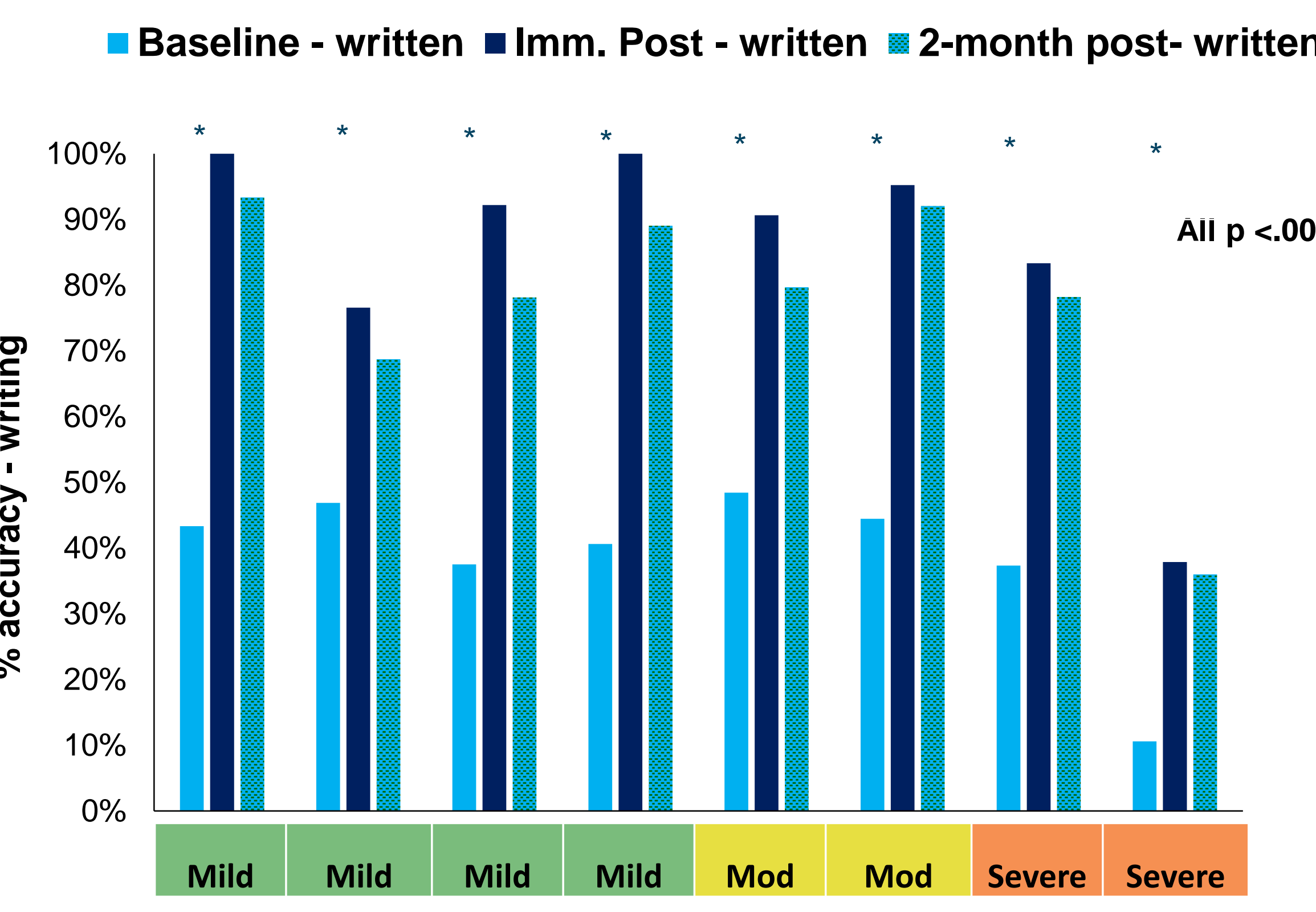

\section{Results - Untrained words}

- No change in performance was observed for untrained words at either the immediate post or the 2-month follow-up assessment.

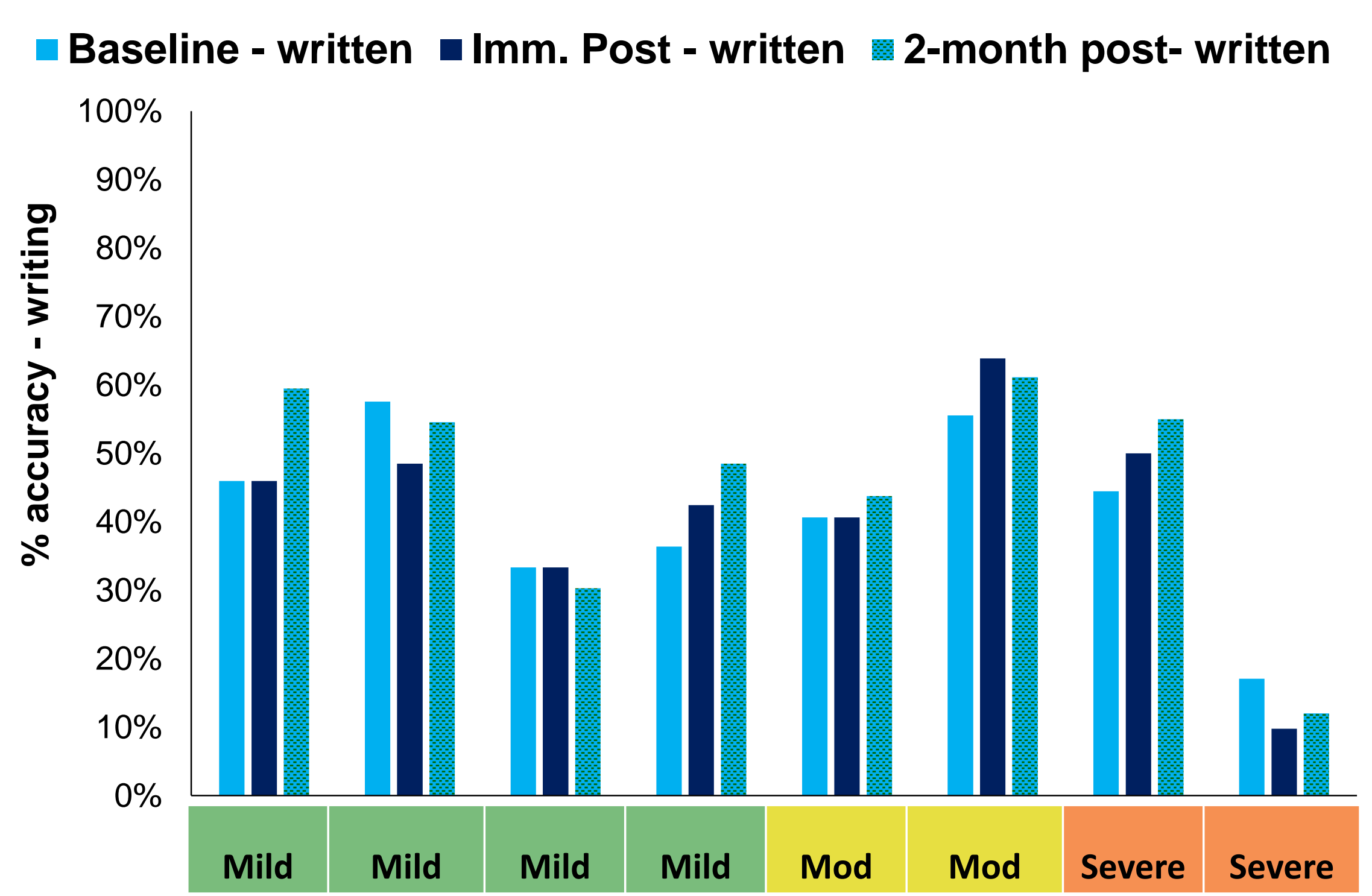

\section{Results - Error type}

- Rate of "don't know" and semantic errors reduced while spelling errors increased for trained words; error rates remained unchanged for untrained words.

- Although mild-moderately impaired patients made fewer errors than the severe patients, error patterns were similar across all participants.

Error Type

\begin{tabular}{|c|c|c|}
\hline $\begin{array}{l}2 \text { Baseline } \\
\text { Errors - } \\
\text { All lists } \\
(\mathrm{n}=486)\end{array}$ & $\begin{array}{c}\Sigma \operatorname{Imm} . P o s t \\
\text { Errors - } \\
\text { Trained } \\
(\mathrm{n}=67)\end{array}$ & $\begin{array}{c}\Sigma \text { Imm.Post } \\
\text { Errors - } \\
\text { Untrained } \\
(n=165)\end{array}$ \\
\hline $225(46 \%)$ & $12(18 \%)$ & $86(52 \%)$ \\
\hline $59(12 \%)$ & $39(58 \%)$ & $22(13 \%)$ \\
\hline $180(37 \%)$ & $7(10 \%)$ & $48(29 \%)$ \\
\hline $15(3 \%)$ & $3(4 \%)$ & $6(4 \%)$ \\
\hline $7(1 \%)$ & $6(9 \%)$ & $3(2 \%)$ \\
\hline
\end{tabular}

Don't Know

Spelling erro

Semantic error

Non-related error

Neologism

\section{Discussion / Conclusions}

- Significant improvements in both spoken and written retrieval can be achieved in SD following an 8-week retraining program.

- These improvements are specific to words that are actively trained; word retraining does not affect untrained words (either positively or negatively).

- Performance is largely retained over the short-term (2-months), although patients with severe semantic deficits are likely to require ongoing revision.

- After training, "don't know" and semantic errors are less common, but spelling errors may occur, particularly for patients with severe impairments.

- A simple home-practice program can benefit people with SD, even in cases of severe semantic impairment.

\section{References}

1) Hodges et al (1999). The differentiation of semantic dementia and frontal lobe dementia (temporal and fronta variants of frontotemporal dementia) from early

Alzheimer's disease: A comparative neuropsychological study. Neuropsychology, 13: 31-40.

2) Croot et al (2009). Impairment- and

activity/participation-directed interventions in progressive language impairment: Clinical and theoretical issues. Aphasiology, 23: 125-160.

3) Savage et al (2013). Bringing words back to mind improving word production in semantic dementia. Cortex. 49(7), 1823-1832.

4) Savage et al (2015). Cognitive intervention in Semantic Dementia: maintaining words over time. Alzheimer Disease and Associated Disorders, 29 (1): 55-62.

5) Savage et al (2013). Distinguishing subtypes in primary progressive aphasia: application of the Sydney language battery. Dementia and Geriatric Cognitive Disorders, 35(34), 208-218. 